\title{
Nanocrystalline and Thin Film Germanium Electrodes with High Lithium Capacity and High Rate Capabilities
}

\author{
J. Graetz, ${ }^{*}$ C. C. Ahn, R. Yazami, and B. Fultz**
}

Division of Engineering and Applied Science, California Institute of Technology, Pasadena, California 91125, USA

Germanium nanocrystals (12 nm mean diam) and amorphous thin films (60-250 nm thick) were prepared as anodes for lithium secondary cells. Amorphous thin film electrodes prepared on planar nickel substrates showed stable capacities of $1700 \mathrm{mAh} / \mathrm{g}$ over 60 cycles. Germanium nanocrystals showed reversible gravimetric capacities of up to $1400 \mathrm{mAh} / \mathrm{g}$ with $60 \%$ capacity retention after 50 cycles. Both electrodes were found to be crystalline in the fully lithiated state. The enhanced capacity, rate capability $(1000 \mathrm{C})$, and cycle life of nanophase germanium over bulk crystalline germanium is attributed to the high surface area and short diffusion lengths of the active material and the absence of defects in nanophase materials.

(C) 2004 The Electrochemical Society. [DOI: 10.1149/1.1697412] All rights reserved.

Manuscript submitted August 18, 2003; revised manuscript received November 6, 2003. Available electronically March 23, 2004.

Binary lithium alloys (Li-X) are currently of interest for anodes in rechargeable electrochemical cells. In addition to their low operating voltage $v s$. lithium $(\sim 300 \mathrm{mV})$, these alloys offer large theoretical energy densities. The largest theoretical gravimetric capacity of the group IV elements ( $\mathrm{Si}, \mathrm{Ge}, \mathrm{Sn}, \mathrm{Pb}$ ) occurs in $\mathrm{Li}-\mathrm{Si}$, which can accommodate up to 4.4 lithium atoms per silicon. Despite the promising energy densities of these alloys, a large volume expansion is associated with lithiation and the resulting stresses tend to decrepitate the host after a few cycles. ${ }^{1-5}$ In addition, the slow kinetics of lithium transport have limited the application of these anodes to medium and high temperature cells using molten electrolytes. ${ }^{6-8}$

Recent investigations into the electrochemical properties of the Li-Si system have demonstrated an improved room temperature cycle life in nanocrystalline and thin film electrodes. ${ }^{5,9-12}$ An analogous system, Li-Ge, has received little attention. The Li-Ge system has a theoretical capacity of $1.6 \mathrm{Ah} / \mathrm{g}\left(\mathrm{Li}_{4,4} \mathrm{Ge}\right)$, which is $40 \%$ of the theoretical Li-Si capacity. On the other hand, silicon typically forms a native oxide on its outermost layers and the presence of a surface oxide reduces the overall capacity. In addition, the silicon oxide reacts with lithium during the initial cycle, forming $\mathrm{Li}_{2} \mathrm{O}$ and elemental silicon, resulting in a large irreversible capacity for the first cycle. The native oxide accounts for a significant fraction of the silicon atoms when the material is in a nanostructured form. In contrast, germanium does not have a strong affinity to form a native oxide and, therefore, the Li-Ge system is a model alloy for investigating the properties of nanostructured electrodes of lithium with group IV elements.

In addition to providing general insights into the $\mathrm{Li}-\mathrm{X}$ system, nanostructured $\mathrm{Li}-\mathrm{Ge}$ may be a viable candidate anode for lithium secondary cells. Although the high cost of elemental germanium has discouraged research efforts on this system in the past, germanium is an abundant element in the earth's crust and the current price is maintained by the lack of demand. In addition to the benefit from minimal native oxide on germanium, the diffusivity of lithium in germanium is $\sim 15$ times greater than that of lithium in silicon at $360^{\circ} \mathrm{C}\left(2.14 \times 10^{-7} \mathrm{~cm}^{2} / \mathrm{s}\right.$ for $\mathrm{Ge}$ and $1.47 \times 10^{-8} \mathrm{~cm}^{2} / \mathrm{s}$ for $\mathrm{Si}){ }^{13}$ The empirical equation for the diffusion coefficient

$$
D=D_{0} \exp [-Q / R T]
$$

where $T$ is the temperature, $Q$ is the activation energy, and $D_{0}$ is a constant prefactor, suggests that the diffusivity of lithium in germanium may be over 400 times greater than that of lithium in silicon at room temperature (using values $Q(\mathrm{~kJ} / \mathrm{mol}) / D_{0}\left(\mathrm{~cm}^{2} / \mathrm{s} \times 10^{7}\right), 64 /$ 0.0023 for silicon and $49 / 0.0025$ for germanium ${ }^{13}$ ).

* Electrochemical Society Student Member.

** Electrochemical Society Active Member.

\section{Experimental}

Electrodes were prepared by two different methods utilizing physical vapor deposition. In the first, evaporation, a charge of elemental germanium was heated resistively in a tungsten wire basket under a vacuum of $2 \times 10^{-6}$ Torr. The material was evaporated at a rate of $\sim 10^{-5} \mathrm{~g} / \mathrm{cm}^{2} / \mathrm{s}$ and deposited directly onto a substrate located near the evaporating basket. In the second method, the material was evaporated at a similar rate $\left(10^{-5} \mathrm{~g} / \mathrm{cm}^{2} / \mathrm{s}\right)$ under a gas flow of $2 \times 10^{-3}$ Torr. A "forming gas" of $90 \% \mathrm{Ar}$ and $10 \% \mathrm{H}_{2}$ was used to reduce the oxygen contamination. In this method, the evaporated atoms cool quickly in the forming gas and thereby nucleate nanoscale crystallites within the gas. The gas-phase ballistic deposition of the entrained nanoparticles produces nanocrystalline aggregates, which are similar to those produced when silicon is ballistically deposited under equivalent conditions. ${ }^{11}$

The germanium electrodes were prepared as-deposited on planar nickel substrates. The substrates were roughened using 400 grit sandpaper to increase the surface area and to improve the electrical contact between the electrode and the current collector. The mass of the deposited material was determined using a Metler micro-balance accurate to $1 \mu \mathrm{g}$. In the interest of a purely fundamental investigation of the electrochemical behavior of nanoscale germanium, no conductive additives or binders were used in the nanostructured electrodes.

To make conventional "control samples," electrodes of bulk germanium were prepared by crushing elemental germanium into a powder of particle size less than $38 \mu \mathrm{m}$. A binder of $10 \mathrm{wt} \%$ polyvinylidene fluoride and a conductive diluent of $8 \mathrm{wt} \%$ acetylene black were added to the powder to improve the cycling characteristics. The electrode powder was pressed into a pellet using a pressure of approximately $90 \mathrm{MPa}$.

The germanium electrodes were cycled in 2016 coin cells using lithium metal as a counter electrode. An equivolume mixture of ethylene carbonate (EC) and dimethyl carbonate (DMC) with $\mathrm{LiPF}_{6}$ was used as the electrolyte and a $0.50 \mathrm{~mm}$ thick strip of fiberglass was used as a separator. A polyethylene separator was used in the bulk germanium cell. The electrochemical tests were performed with an Arbin Instruments BT2000 battery cycler.

Transmission electron microscopy (TEM) was performed in a Philips EM 420 operated at $100 \mathrm{kV}$. The TEM samples were prepared by physical vapor deposition onto a holey carbon grid. X-ray diffraction (XRD) was performed with an Inel CPS-120 diffractometer using Co $\mathrm{K} \alpha$ radiation $(\lambda=1.790 \AA)$. The as-deposited samples were prepared on glass substrates and the diffraction data were acquired in air. The lithiated electrodes were prepared by discharging an electrochemical cell to a potential of $0 \mathrm{~V}$. The electrodes were extracted from the cells in an argon glove box, where the specimens were mounted on glass and covered with Kapton to prevent air contamination. The amorphous background produced by the 


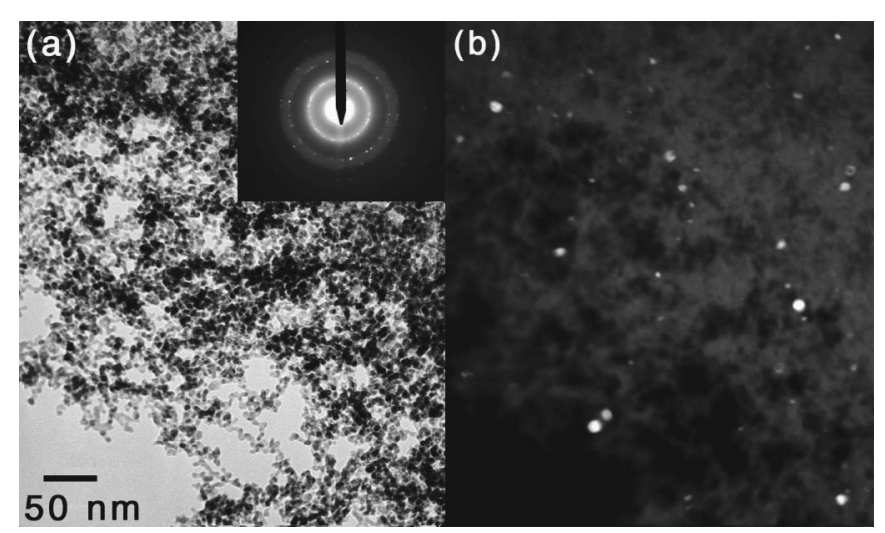

Figure 1. (a) Bright-field and (b) dark-field TEM images of ballistically deposited germanium showing an agglomeration of nanocrystals. The electron diffraction pattern is displayed in the inset.

sample holder (e.g., glass and Kapton) was measured and subtracted from each of the diffraction patterns.

\section{Results}

The two deposition methods generated unique films with distinct microstructures. Bright and dark-field TEM images of the germanium film prepared by ballistic consolidation are displayed in Fig. $1 \mathrm{a}$ and $1 \mathrm{~b}$. The dark-field images were created using the (111) diffraction ring. The film consists of a web of interconnected nanocrystalline germanium with a mean particle diameter of $10 \mathrm{~nm}$. The distinct bright spots in the electron diffraction pattern displayed in the inset of Fig. 1 also indicate a crystalline structure.

The microstructure of the evaporated germanium was dissimilar to that of the ballistically deposited material. When evaporated in high vacuum, the germanium formed a continuous thin film as seen in the bright-field and dark-field TEM images displayed in Fig. 2a and $b$. The broad rings of the electron diffraction pattern displayed in the inset of Fig. 2a suggest the material is entirely amorphous. In the middle of the film the material appears to be contiguous and uniform, while the material on the edge of the carbon substrate appears to have a columnar structure. It is likely that this structure is not representative of the entire film and is simply an artifact of the substrate edge.

Figure 3a displays the XRD patterns from ballistically deposited germanium (as-deposited), the electrochemically lithiated germanium, and the electrochemically delithiated germanium. The peak positions of the as-deposited material are consistent with the diamond cubic structure with a lattice parameter of $a=5.66 \AA$. The

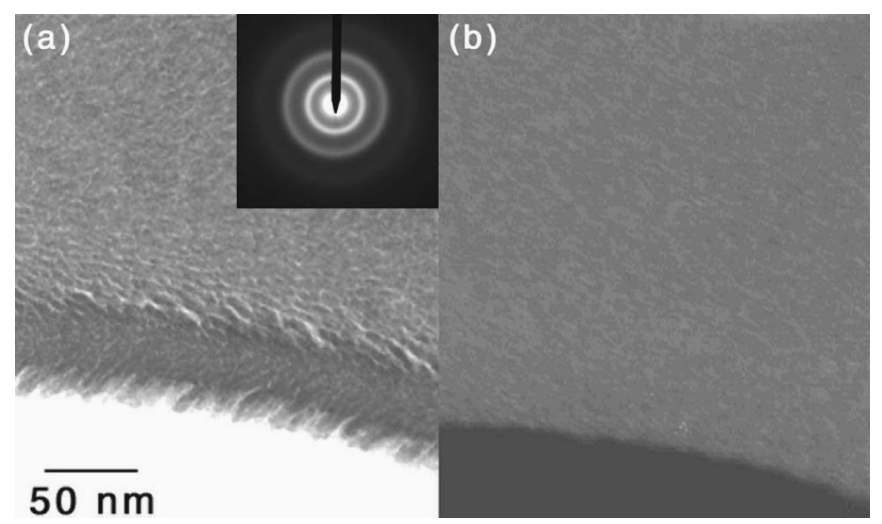

Figure 2. (a) Bright-field and (b) dark-field TEM images of evaporated germanium showing a uniform amorphous thin film. The electron diffraction pattern is displayed in the inset.

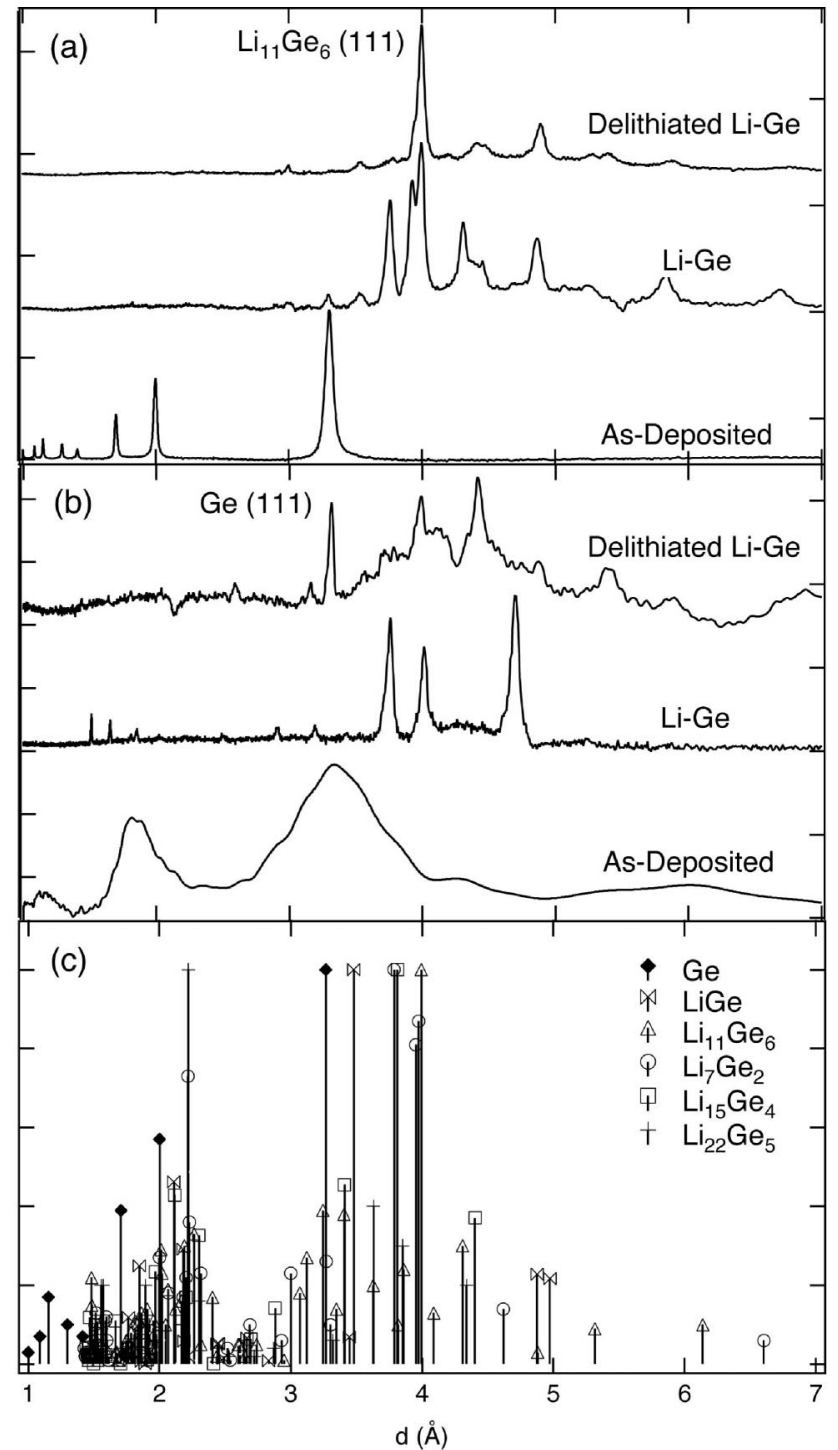

Figure 3. XRD patterns of the as-deposited, electrochemically lithiated, and electrochemically delithiated electrodes of (a) ballistically deposited and (b) evaporated germanium. (c) Peak positions of pure germanium and a number of Li-Ge phases. ${ }^{15,18-20}$

broad peaks of the as-deposited material are indicative of a small crystallite size, which was estimated using the approximate Scherrer equation ${ }^{14}$ to be $12 \mathrm{~nm}$. The XRD pattern from the ballistically deposited material after electrochemical lithiation contains a number of Bragg peaks at large $d$-spacings, indicating that the material is at least partially crystalline. Large interplanary spacings are expected for most of the Li-Ge phases. ${ }^{15-20}$ The electrochemically delithiated material appears to be predominately $\mathrm{Li}_{11} \mathrm{Ge}_{6}$, suggesting that not all of the lithium is removed from the nanocrystalline electrode. The XRD patterns of the evaporated material are displayed in Fig. 3b. The broad peaks of the as-deposited material confirm that the material is initially amorphous, while the sharp peaks from the lithiated electrode suggest that the material contains some crystalline Li-Ge phases. A strong Ge (111) diffraction peak is observed in the upper trace of Fig. 3b, indicating that the material returns to a predominately germanium phase upon electrochemical delithiation. The structure at 4.0 and $4.4 \AA$ may be indicative of some residual lithiated phase (such as $\mathrm{Li}_{11} \mathrm{Ge}_{6}$ ). The patterns of Fig. $3 \mathrm{~b}$ also suggest that the evaporated amorphous material becomes crystalline upon lithiation and remains crystalline upon the subsequent delithiation. 


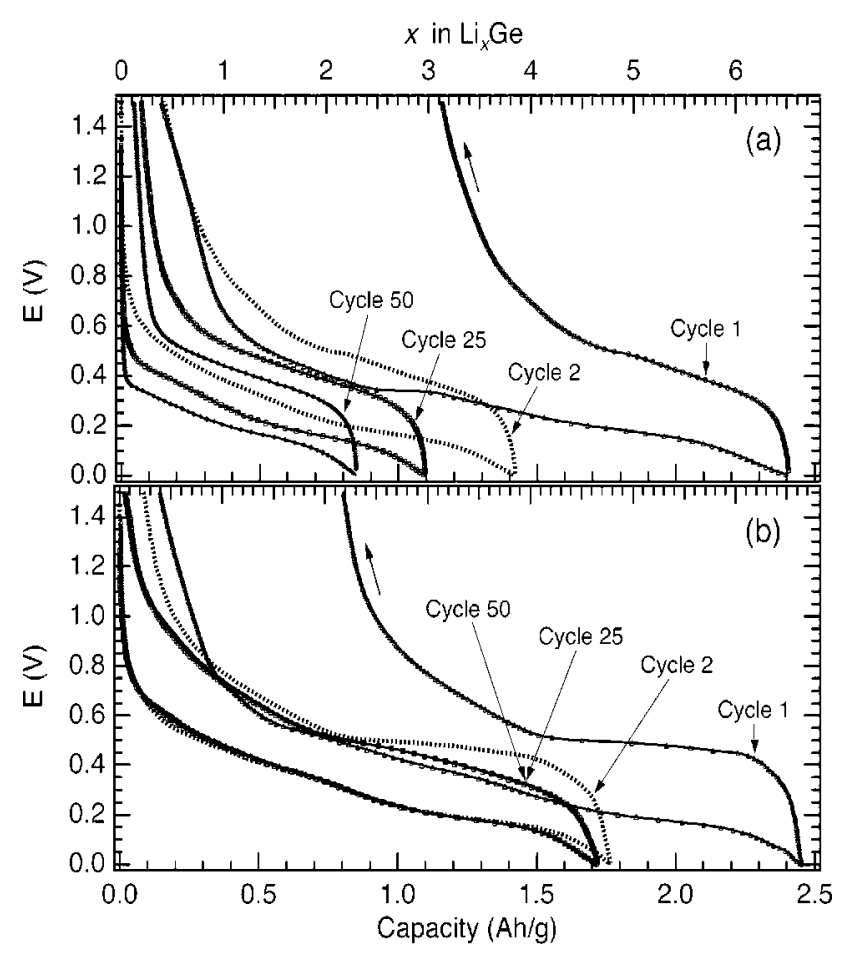

Figure 4. (a) Voltage profiles from ballistically deposited germanium and (b) evaporated germanium cycled at a rate of $\mathrm{C} / 4$. The arrows indicate the charge step of the first cycle.

The peak positions from elemental germanium as well as a number of Li-Ge phases ${ }^{15,18-20}$ are shown in Fig. 3c.

A plot of the voltage profiles from a $236 \mu \mathrm{g}$ electrode of ballistically deposited nanocrystalline germanium and a $42 \mu \mathrm{g}$ electrode of an evaporated germanium nanofilm are shown in Fig. 4 . The most salient feature is the high reversible capacity of the nanostructured electrodes. The evaporated germanium nanofilm accommodates approximately 4.5 lithium atoms per germanium atom, which is slightly higher than the theoretical limit for crystalline $\mathrm{Li}_{22} \mathrm{Ge}_{5}$. The film thickness is estimated to be $60 \mathrm{~nm}$ based upon the electrode mass and bulk material density. Thicker films of up to $250 \mathrm{~nm}(180$ $\mu \mathrm{g})$ were cycled at slower rates with similar results. The ballistically deposited material hosts up to 3.8 lithium atoms per germanium atom. Graphs of the differential capacity, $\mathrm{d}|x| / \mathrm{dE}$, are displayed in Fig. $5 \mathrm{a}$ and $\mathrm{b}$, where $x$ is the lithium concentration in $\mathrm{Li}_{x} \mathrm{Ge}$ and $\mathrm{E}$ is the cell potential. The peaks are indicative of lithium insertion into equipotential sites. The dual peaks exhibited in these plots suggest that at least two new phases are formed during lithiation. Upon lithium insertion/extraction these new phases are formed at 180/380 $\mathrm{mV}$ and $360 / 500 \mathrm{mV}$. The disparity in potentials between lithium insertion and extraction is due to the over potential resulting from the constant current, or nonequilibrium state. The actual phase transition energy can be approximated by averaging the charge and discharge values, giving $\sim 280$ and $\sim 430 \mathrm{mV}$ at room temperature.

Plots of the cycle life for bulk crystalline germanium (grain size $\leqslant 38 \mu \mathrm{m}$ ) and the two types of nanostructured germanium electrodes are displayed in Fig. 6 . The nanostructured electrodes were cycled at a rate of approximately $\mathrm{C} / 4$ (i.e., $375 \mathrm{~mA} / \mathrm{g}$ ) between 0 and $1.5 \mathrm{~V}$, whereas the bulk electrode was cycled at a much slower rate $(\sim \mathrm{C} /$ 30) to maximize the specific capacity. Despite the gentle cycling conditions, bulk germanium exhibited a poor cycle life, with nearly complete capacity loss by the seventh cycle. The evaporated germanium nanofilm exhibits a large first-cycle irreversible capacity with a steady specific capacity of $1.7 \mathrm{Ah} / \mathrm{g}$ and no detectable capacity fade over 60 cycles. A similar first-cycle capacity loss is observed with the ballistically deposited germanium. Although the initial stable

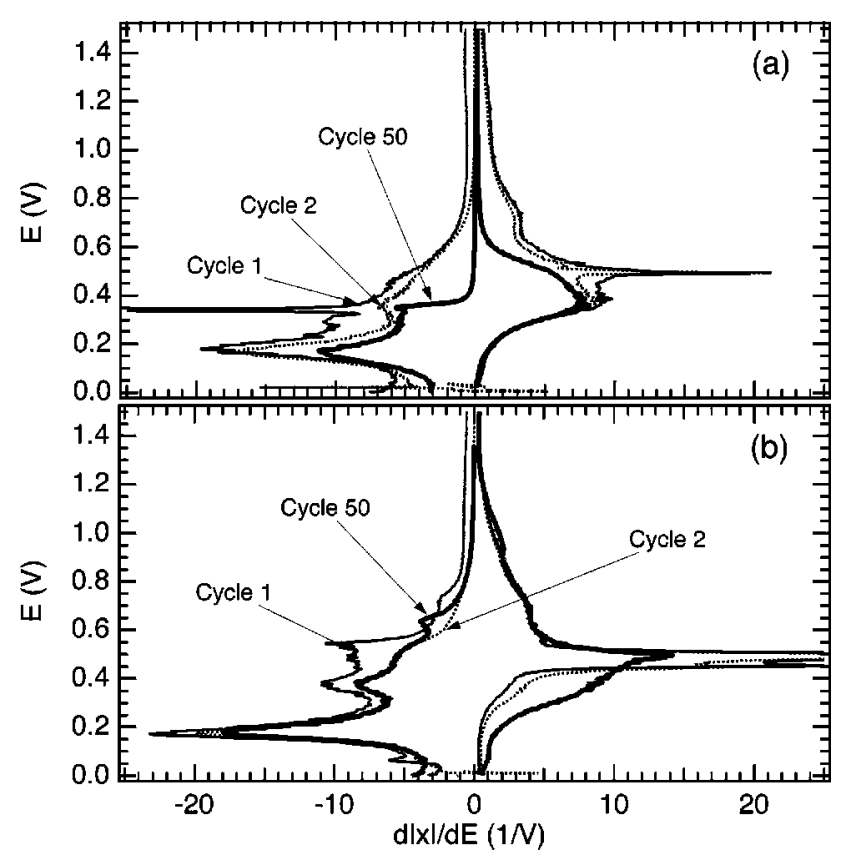

Figure 5. (a) Differential capacities from ballistically deposited germanium cycled at a rate of C/4. (b) Differential capacities from evaporated germanium.

capacity is similar to that of the evaporated nanofilm $(\sim 1.4 \mathrm{Ah} / \mathrm{g})$, the ballistically deposited electrode exhibits a constant capacity fade of approximately $0.01 \mathrm{Ah} / \mathrm{g}$ per cycle.

The rate capabilities of the nanostructured electrodes were also investigated. A $250 \mathrm{~nm}$ evaporated germanium film $(180 \mu \mathrm{g})$ was cycled at a constant discharge rate of $0.5 \mathrm{C}$ and a variable charge rate from $0.5 \mathrm{C}$ to $1000 \mathrm{C}$. At $0.5 \mathrm{C}$ the cell was cycled between 0 and $1.5 \mathrm{~V}$ and the upper limit of the potential was increased by $50-100 \mathrm{mV}$ on each subsequent cycle to account for the over potential associated with the increased cycling rates. A ballistically deposited nanocrystalline film $(314 \mu \mathrm{g})$ was cycled under similar conditions. Figure 7 shows a plot of the normalized capacity $\left(Q / Q_{0}\right)$ at various discharge rates. The vertical error bars reflect the uncertainty

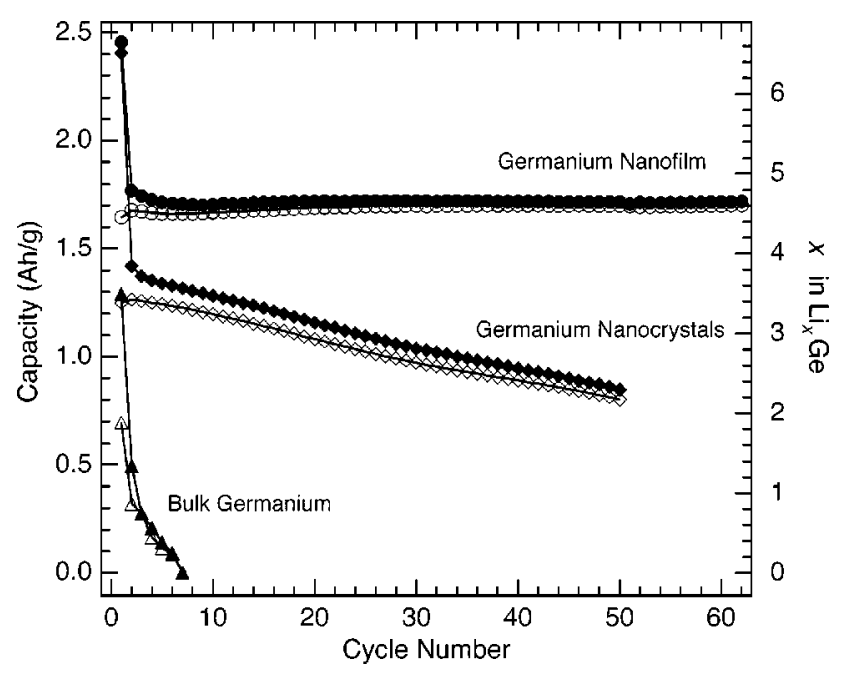

Figure 6. Cycle life of germanium from ballistically deposited nanocrystals, an evaporated amorphous nanofilm, and the control sample of bulk crystalline germanium. The light and shaded markers represent the charge and discharge cycles, respectively. 


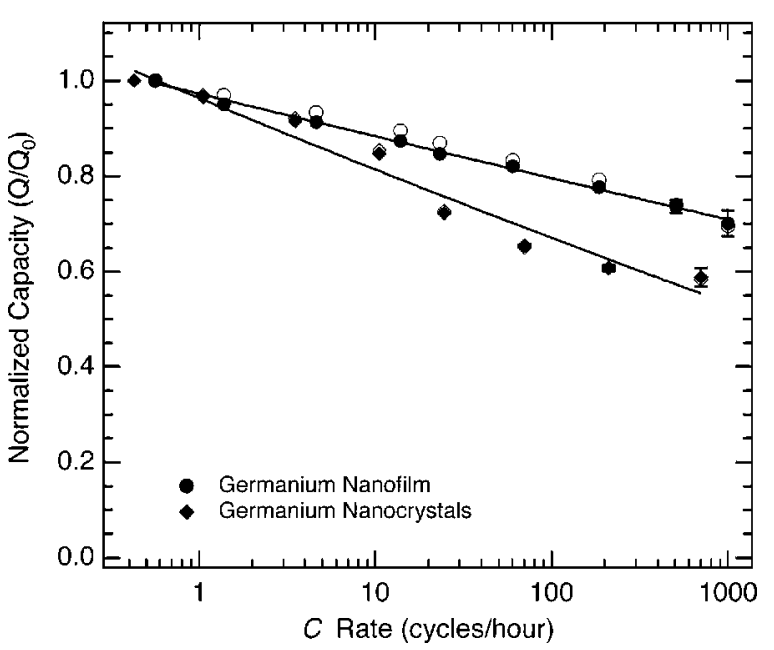

Figure 7. Rate capabilities of ballistically deposited germanium and an evaporated thin film. The electrodes were lithiated (discharged) at a constant rate of $1 \mathrm{C}$ and delithiated (charged) at a variable rate. The light and shaded markers represent the charge and discharge cycles, respectively.

associated with the data acquisition system and are visible only when the charging times are short. Remarkably, only a moderate capacity loss is observed between 1 and $1000 \mathrm{C}$.

A test of the cycle life at high rates was also performed on the amorphous thin film electrode. In this experiment the $250 \mathrm{~nm}$ germanium film was cycled at a discharge rate of $0.5 \mathrm{C}$ and a charge rate of $1000 \mathrm{C}$. The cell was cycled between 0 and $3.0 \mathrm{~V}$. Figure 8 displays a plot of the charge and discharge capacities over 30 cycles. This figure clearly illustrates the overlap between the high-rate charge step and low-rate discharge step. This indicates that all of the lithium inserted over a $4 \mathrm{~h}$ lithiation period is removed in less than $4 \mathrm{~s}$ upon delithiation.

\section{Discussion}

Li-Ge phases.-According to the Li-Ge phase diagram, a number of phase transitions are expected to occur during lithiation at room temperature. ${ }^{15-20}$ Phase transitions are easily identified by plateaus in the voltage profile (Fig. 4). The Gibbs phase rule prohibits any variation in the chemical potential (or cell voltage) at fixed temperature when two simultaneous phases are present. In a two-phase re-

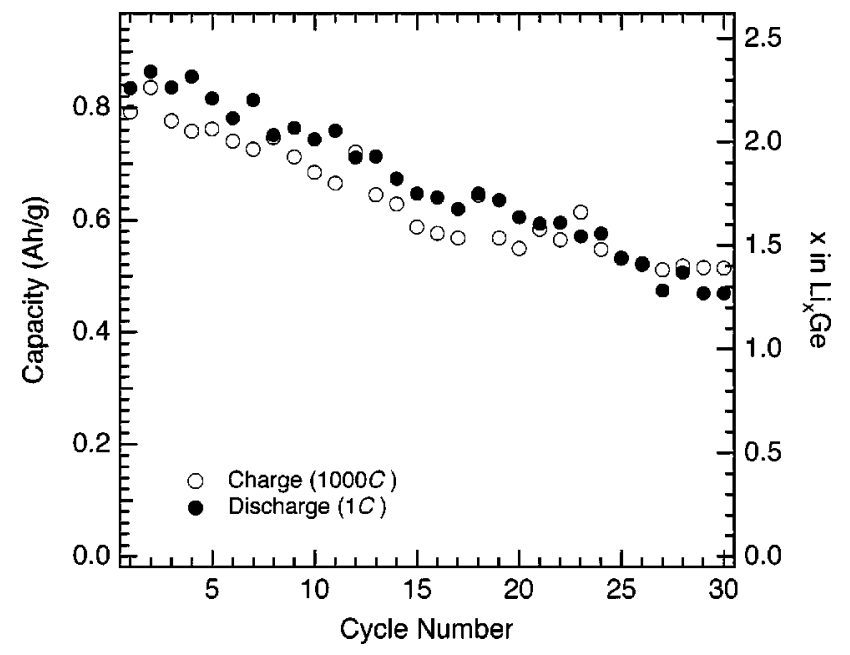

Figure 8. Cycle life of thin film amorphous germanium at a lithiation rate of $1 \mathrm{C}$ and a delithiation rate of $1000 \mathrm{C}$. The light and shaded markers represent the charge and discharge cycles, respectively. gion, the potential is constant during changes in lithium concentration as one phase grows at the expense of the other. The voltage profiles of Fig. 4a and b exhibit a reasonably smooth slope on the charge and discharge cycles for both of the nanostructured electrodes. However, even subtle variations in the slope may be indicative of the formation of a new phase. The differential capacity plots of Fig. 5a and b were prepared to accentuate changes in the slope of the potential curves. Peaks in the differential capacity indicate regions of the potential where lithium ions are entering nearly equipotential sites. The presence of multiple peaks suggests that a number of different Li-Ge phases are formed during electrochemical lithiation in both the evaporated and ballistically-deposited electrodes. This is in contrast to silicon, which forms amorphous phases upon lithiation at room temperature. ${ }^{21}$

The peaks in the Li-Ge diffraction patterns of Fig. 3a and b correspond to a number of crystallographic phases, suggesting that the material is heterogeneous in the lithiated state. The most obvious phases present are $\mathrm{LiGe}^{15} \mathrm{Li}_{7} \mathrm{Ge}_{2},{ }^{18}$ and $\mathrm{Li}_{15} \mathrm{Ge}_{4} \cdot{ }^{19}$ Other phases that may be present in small quantities are $\mathrm{Li}_{11} \mathrm{Ge}_{6},{ }^{16} \mathrm{Li}_{9} \mathrm{Ge}_{4},{ }^{17}$ and $\mathrm{Li}_{22} \mathrm{Ge}_{5} \cdot{ }^{20}$ In addition, the broad underlying peaks located at 2.2 and $4.5 \AA$ suggest that there may be an amorphous phase present in the lithiated material. However, unlike the silicon system, germanium does not appear to become amorphous during electrochemical lithiation at room temperature. In fact, electrochemical lithiation appears to enhance the crystallinity of the material, which may be attributed to the rapid rate kinetics of lithium in germanium.

Surface-electrode interphase.-The large irreversible capacity observed on the initial cycle is likely attributed to the formation of a surface-electrolyte interphase (SEI). A reaction of lithium with the electrolyte accompanies the initial lithiation of the germanium electrode, forming a passivation layer. This reaction is beneficial when using a carbon electrode because it prevents solvent decomposition and cointercalation during lithiation. Although the lithium alloy electrodes are typically not affected by solvent cointercalation, the presence of a passivation layer may prevent spontaneous solvent decomposition. Despite the first cycle capacity loss, the growth of the SEI layer does not appear to be detrimental to the specific capacity or cycle life. The first cycle capacity loss is $\sim 70 \%$ of what was observed in nanostructured Li-Si materials. ${ }^{11}$ The lower firstcycle irreversible capacity may be attributed to the lack of a native oxide on germanium, which might otherwise contribute to the SEI if it were reduced by lithium.

Cycle life.-The high specific capacities of the nanostructured germanium electrodes were found to be stable for over 50 cycles (Fig. 6). Although the high capacities are expected from the high solubility of lithium in germanium, the complete lithiation of germanium has never been observed at room temperature on these time scales. Similarly, the slow kinetics of lithium in germanium are expected to create large stresses within the material, causing the decrepitation of the host. This is clearly observed in the attenuated cycle life of the bulk germanium electrode (Fig. 6). Remarkably, there is no capacity loss observed in the amorphous nanofilm over 62 cycles. Similarly, the nanocrystalline electrode exhibits only a slow loss of capacity over 50 cycles. These results indicate that the active germanium particles do not decrepitate significantly during electrochemical cycling. The constant capacity loss observed in the nanocrystalline system is attributed to the spallation of particles off the surface of the current collector resulting from changes in the sample volume by up to $230 \%$ during cycling. ${ }^{22}$

The stability of the amorphous nanofilm during cycling is surprising. Although this electrode is thin, suggesting rapid lithium transport perpendicular to the film, the electrode is attached to a rigid substrate. The large volume expansion that occurs during lithiation is expected to create large strain gradients as the lithium front propagates in and out of the film. Such strains are more than sufficient to debond the film from the substrate. However, complete decohesion of the film does not occur during cycling; the film remains electrically intact. Despite the electrical continuity, it is likely 
that cracks are generated during the initial cycling and the propagation of these cracks partitions the film into isolated islands. It is believed that these islands are able to accommodate the volume changes of cycling while maintaining contact with the current collector. Although the film is broken, the cell does not lose capacity because the material does not decrepitate or spall off the surface as it does for electrodes made of bulk germanium.

The cycling stability of lithium in the ballistically deposited germanium is easier to understand. The reversibility is attributed to the absence of conventional mechanisms for microstructural damage in nanoscale materials. ${ }^{11}$ The formation and propagation of cracks is prohibited due to the absence of dislocations and the large critical flaw size for crack growth, with respect to the particle diameter. The mitigation of particle decrepitation is also attributed to the short timescales required for the relaxation of diffusional stresses in nanostructured materials. The relaxation time is proportional to the square of the diffusion distance. The diffusion of lithium into the host distorts the lattice parameter, $a$, to approximately $3^{1 / 3} a$. The strain is not intrinsically harmful to the host, but rather it is the gradients in strain that are responsible for breaking apart the electrode during cycling. When the diffusion lengths are short, the relaxation times are short and the material is strained uniformly.

The reversible cycling of 4.5 lithium atoms per germanium atom in the amorphous thin film and 3.8 lithium atoms per germanium atom in the nanocrystalline film are considerably larger than the reversible capacities measured in analogous nanocrystalline silicon materials. Silicon electrodes prepared and cycled under similar conditions exhibited reversible capacities of 2.1 lithium atoms per silicon atom in the amorphous thin film and 1.1 lithium atoms per silicon atom in the nanocrystalline film. ${ }^{11}$ The larger reversible lithium uptake in the germanium system is attributable in part to the higher diffusivity, $D$, of lithium in germanium at room temperature $\left(D_{\mathrm{Ge}} \approx 400 D_{\mathrm{Si}}\right)$. In addition, the $25 \AA$ native oxide on the surface of the silicon electrode reduces the overall specific capacity, increases the first cycle irreversible capacity, and contributes to the SEI, which ultimately increases the cell impedance.

Rate capabilities.-High rate capabilities are expected in nanostructured electrodes due to short relaxation times. For solid state diffusion, the time required for a system to relax is proportional to the square of the diffusion distance (i.e., particle diameter or film thickness). Therefore, diffusion through a $10 \mathrm{~nm}$ particle will occur 10,000 times faster than through a $1 \mu \mathrm{m}$ particle. The high rate capabilities of nanostructured electrodes are well documented and cycling rates of up to $4000 \mathrm{C}$ have been demonstrated in vanadium oxide. ${ }^{23}$ Therefore, it is not surprising that the results of Fig. 7 and 8 indicate high rate capabilities in the nanostructured germanium electrodes. The capacities measured at variable charging rates (Fig. 7) indicate a slow exponential (linear on a log scale) capacity decay up to $1000 \mathrm{C}$. There is no indication of a catastrophic loss of capacity typically associated with an electrochemical cell limited by solid state diffusion. The voltage profiles at $1000 \mathrm{C}$ are similar to those at $0.5 \mathrm{C}$, indicating that the lithium is alloyed with the host and not simply plated on the electrode surface. Although the capacities at $1000 \mathrm{C}$ are relatively high $(500-800 \mathrm{mAh} / \mathrm{g})$, the capacity fades by approximately $35 \%$ over 30 cycles. This capacity loss may be attributed to some decrepitation of the electrode during cycling under these extreme conditions.

\section{Conclusion}

Electrodes of nanostructured elemental germanium formed alloys of $\sim 80$ atom\% lithium. Although this composition is consistent with the $\mathrm{Li}_{22} \mathrm{Ge}_{5}$ phase in crystalline Li-Ge, these materials exhibited a number of different crystallographic phases when lithiated. A high initial specific capacity was measured in a control sample of bulk germanium. However, the complete loss of capacity after only a few cycles suggests that large particles decrepitate during cycling as a result of the large alloying strains. In contrast, thin films of amorphous germanium exhibited capacities of $1.7 \mathrm{Ah} / \mathrm{g}$ with no capacity loss over 62 cycles. Electrochemically lithiated nanocrystalline germanium demonstrated capacities of up to $1.4 \mathrm{Ah} / \mathrm{g}$ with an $\sim 60 \%$ capacity retention after 50 cycles. The high reversibility is attributed to the nanoscale microstructure of these electrodes. The short diffusion paths reduce strain fields and mitigate microstructural cycling damage. The short diffusion distances are also responsible for the high rate capabilities of these electrodes, which exhibited rates of up to $1000 \mathrm{C}$ with only a moderate reduction of capacity. These results suggest that nanoscale microstructures can be used to increase diffusion rates and reduce particle decrepitation in lithium alloy electrodes.

\section{Acknowledgment}

This work was supported by the Department of Energy through Basic Energy Sciences Grant no. DE-FG03-00ER15035.

The authors assisted in meeting the publication costs of this article.

\section{References}

1. J. O. Besenhard, J. Yang, and M. Winter, J. Power Sources, 68, 87 (1997).

2. M. Winter, J. O. Besenhard, M. E. Spahr, and P. Novak, Adv. Mater. (Weinheim, Ger.), 10, 725 (1998).

3. J. Yang, M. Winter, and J. O. Besenhard, Solid State Ionics, 90, 281 (1996).

4. M. Winter and J. O. Besenhard, Electrochim. Acta, 45, 31 (1999).

5. S. Bourderau, T. Brousse, and D. M. Schleich, J. Power Sources, 81, 233 (1999).

6. R. A. Sharma and R. N. Seefurth, J. Electrochem. Soc., 123, 1763 (1976).

7. C. J. Wen and R. A. Huggins, J. Solid State Chem., 37, 271 (1981).

8. M. R. StJohn, A. J. Furgala, and A. F. Sammells, J. Electrochem. Soc., 127, C136 (1980).

9. H. Li, X. Huang, L. Chen, Z. Wu, and Y. Liang, Electrochem. Solid-State Lett., 2, 547 (1999).

10. G. W. Zhou, H. Li, H. P. Sun, D. P. Yu, Y. Q. Wang, X. J. Huang, L. Q. Chen, and Z. Zhang, Appl. Phys. Lett., 75, 2447 (1999).

11. J. Graetz, C. C. Ahn, R. Yazami, and B. Fultz, Electrochem. Solid-State Lett., 6, A194 (2003).

12. S. Ohara, J. Suzuki, K. Sekine, and T. Takamura, J. Power Sources, 119-121, 591 (2003).

13. C. S. Fuller and J. C. Severiens, Phys. Rev., 96, 21 (1954).

14. B. Fultz and J. Howe, Transmission Electron Microscopy and Diffractometry of Materials, Springer-Verlag, New York (2000).

15. E. Menges, V. Hopf, H. Schaefer, and A. Weiss, Z. Naturforsch. B, 24, 1351 (1969).

16. U. Frank and W. Muller, Z. Naturforsch. B, 30, 313 (1975).

17. V. Hopf, H. Schafer, and A. Weiss, Z. Naturforsch. B, 25, 653 (1970).

18. V. Hopf, Z. Naturforsch. B, 27, 1157 (1972).

19. Q. C. Johnson, G. S. Smith, and D. Wood, Acta Crystallogr., 18, 131 (1965).

20. E. I. Gladyshevskii, G. I. Oleksiv, and P. I. Kripyake, Sov. Phys. Crystallogr., 9, 269 (1964).

21. P. Limthongkul, Y. I. Jang, N. Dudney, and Y. M. Chiang, Acta Mater, 51, 1103 (2003).

22. R. Nesper, Prog. Solid State Chem., 20, 1 (1990).

23. J. Patrissi and C. R. Martin, J. Electrochem. Soc., 148, A1247 (2001). 